\title{
A Survey on QoS Enhancement in Mobile Multimedia Services using Cross-Layer Design in 4G Wireless Networks
}

\author{
M.Bhuvaneswari \\ Assistant Professor \\ Electronics and Communication Engineering \\ Anna University of Technology, Tiruchirappalli \\ Tamilnadu, India
}

\author{
P.Seethalakshmi \\ Director \\ Centre for Academic Exellence, \\ Anna University of Technology, Tiruchirappalli \\ Tamilnadu, India
}

\begin{abstract}
Present 3G capability to cater for subscriber demand for high data rate and QoS [Quality of Service] is substantially less than $4 \mathrm{G}$ networks. It is expected that $3 \mathrm{G}$ networks will not be able to meet the needs of services like video-conferencing, full motion video etc in terms of QoS. 4G networks provide subscriber with a higher bandwidth $(5 \mathrm{GHz})$, and mobile data rate greater than $100 \mathrm{Mbps}$. However all these could be achieved along with interoperability and seamless mobility with high QoS in a single hand held terminal in 4G heterogeneous environment by using Cross-Layer Feedback Design. The conventional ISO-OSI Layered architecture is not well suited for mobile multimedia application due to the varying nature of wireless channel. In order to cope up the problems of layered architecture, cross-layer design is used in $4 \mathrm{G}$ architecture to facilitate interaction among layers for better adaptability of resources as per users demand. This paper provides past accomplishment and future avenues related to enhancing QoS in $4 \mathrm{G}$ networks.
\end{abstract}

\section{Keywords}

4G networks;Cross-layer feedback design;Quality of Service.

\section{INTRODUCTION}

The increasing demand for multimedia applications with higher data rate led to the development of $4 \mathrm{G}$ mobile networks which promises to have higher data rates than the present data rate $(2-10 \mathrm{Mbps}) .4 \mathrm{G}$ (or) NGN (next generation networks) allows connectivity anytime, anywhere with QoS and mobility functions. QoS is required to deploy real-time communications over IP networks using Cross Layer feedback in 4G. Due to highly variable nature of wireless links and resource poor nature of mobile devices, interaction between layers is done using cross layer feedback to optimally select QoS parameters such as modulation scheme from PHY layer, FEC scheme from MAC layer, required routing protocol from IP layers and so on as the demand arises from user. In this paper, survey is made on all these layers and their specific QoS parameters affecting the data rate of the network. In section 1, Cross-layer concept in Wireless Networks along with QoS in mobile multimedia data is discussed. Section2 deals with Past and on going Research work on QoS and finally section 3 deals with conclusion followed by references.

\section{CROSS- LAYER CONCEPT IN WIRELESS NETWORKS}

Cross-Layer Design allows communication to take place even between nonadjacent layers through additional entities introduced into the system architecture. In cross-layer approach physical layer information is passed to higher layers and vice versa unlike ISO-OSI independent protocol stack providing interaction only with adjacent layer. However, ISO-OSI layer provide interoperability among multi-vendor entities in wireless network but, during data delivery due to unpredictable nature of wireless interface ie., Multi path propagation,shadowing and frequency selective fading etc., pose a serious degradation on acceptable QoS, particularly in a resource limited mobile environment. So, a shift from traditional layering paradigm to Cross-layer coordination across nonadjacent layer is required in NGMN. There are two approaches for Cross-Layer feedback in wireless networks from literature.

i) Top-Down approach:

In top-down approach information will be flowing

downwards to any layer below it.

ii) Bottom-Up approach:

In bottom-up approach information will be flowing upwards to any layer above it.

\subsection{Cross-Layer Approach to Enhance QoS}

From Fig.1, as per user demand, upper layer such as Application layer interacts with lower layers such as Physical \& MAC Layer if needed, to guarantee acceptable QoS. If the user prefers multimedia data download in mobile terminal, the application layer alters the throughput as per demand, by interacting with Network layer for reduced hand-off latency if the data is from heterogeneous network, then with MAC layer for error free transmission and minimum packet loss during delivery and also negotiates with Physical layer to adapt its Modulation / Coding technique by using adaptive modulation or SDR [software Defined Ratio] along with minimum BER [Bit Error Rate]. So, Cross layer feedback is used to make the layers adapt themselves according to the demand in delivery. For instance, if the data is a real time data, then Application layer can interact with Transport layer for minimum packet loss by altering the window size of the receiver. However, by using vertical hand off in Network layer, FEC and Hybrid ARQ in MAC layer and finally, adapting the transmission power, modulation scheme a better / achievable QoS could be provided to the users. 


\subsection{Parameter Abstraction}

Necessary state information is collected from application layer and radio link layer for specific QoS. The transformation of layer specific parameters into parameters that are comprehensible for CL [Cross-Layer] optimizer is done to provide compatibility and signaling over head along with stability.

\subsection{Data link and PHY Parameters}

In Physical layer transmit power, channel estimation, synchronization, signal shaping, modulation and signal detection are QoS parameters of interest.

In Data link layer radio resource allocation (multiuser scheduling or queuing), error control by channel coding, FEC and ARQ are parameters of interest and important.

\subsection{Application Layer Parameter}

In Application layer Compression, Packetization and Transmission Scheduling are important QoS parameters.

\subsection{QoS in Protocol Layers}

Two main challenges need to be addressed in $4 \mathrm{G}$ networks. The first concerns accessibility to different types of cellular network. The second concerns how to maintain the desired end-to-end QoS for traffic that has varying requirements of bandwidth, bit rate, channel characteristics, and especially the handover delay which causes a drop in QoS level. Hence few QoS parameters from literature for Cross layer feedback with ISO-OSI layers is discussed.

Table 1 Cross-Layer parameters for QoS

\begin{tabular}{|c|c|c|}
\hline Layer & $\begin{array}{c}\text { Cross-Layer } \\
\text { parameter for } \\
\text { QoS }\end{array}$ & QoS Levels \\
\hline Physical & $\begin{array}{c}\text { Channel } \\
\text { condition,BER, } \\
\text { transmit } \\
\text { power,coding } \\
\text { and modulation. } \\
\text { Link / }\end{array}$ & $\begin{array}{c}\text { Acceptable } \\
\text { QoS applies } \\
\text { to } \\
\text { jeltay and } \\
\text { packet loss. } \\
\text { rate. }\end{array}$ \\
\hline MAC & $\begin{array}{c}\text { Transaction } \\
\text { level QoS. }\end{array}$ \\
\hline Network & $\begin{array}{c}\text { Handoff latency } \\
\text { Circuit level } \\
\text { QoS }\end{array}$ \\
\hline Transport & Packet loss & $\begin{array}{c}\text { User level } \\
\text { QoS. }\end{array}$ \\
\hline $\begin{array}{c}\text { Applicati } \\
\text { on }\end{array}$ & Throughput & $\begin{array}{c}\text { End to End } \\
\text { QoS. }\end{array}$ \\
\hline
\end{tabular}

\subsubsection{Physical Layer - Interaction with upper layers}

The function of the Physical layer is to transmit raw bits at a certain power and transmission range with reduced bit errors. From [2], in Physical layer the main QoS parameters are BER and transmit power based on channel status. Apart from providing interaction with the upper layers, Physical layer can adapt its Modulation/Coding based on QoS requirements of higher layers.

\subsubsection{Link / MAC Layer}

MAC layer is responsible for error-free transmission over wireless network. It can instruct the PHY layer to increase the transmit power to get reduced BER for error correction at link layer. This kind of increasing transmit power will provide reliability at the expense of more power consumption.

From [5], Link layer QoS parameters are acceptable delay and packet loss reflecting the channel conditions. The Link reliability can be further improved by using FEC \& ARQ specifically hybrid ARQ. Higher layers make use of FEC scheme of Link layer to find the number of frames retransmitted, frames length, when the wireless medium is available for transmission \& hand-off initiation / completion events.

\subsubsection{Network Layer}

The BER of Physical layer can be used by network layer to change to another interface as and when required.

\subsection{Cross-Layer Design in 4G Mobile Networks}

4G Mobile Networks offer users very rich application contents ranging from high-speed data Internet to real-time video conferences and so forth.One of the most important features in $4 \mathrm{G}$ mobile networks is the domination of high-speed packet transmissions or burst traffic in channels. The high-speed burst traffic carried in $4 \mathrm{G}$ mobile networks will exert profound impact on upper-layer design philosophy. The recent study has indicated that traditional seven layer network architecture based on OSI model may not be well suited for $4 \mathrm{G}$ mobile networks, where transactions of short packets will be the major part of the traffic in the channel.

Consider the scenario where a base station (BS) will receive huge number of short bursts from different mobiles situated in different locations in a cell. The BS receiver should be able to respond to the arrival of each packet in a very timely manner. As the packets from different mobiles carry completely different channel characteristics, the receiver should execute all necessary algorithms such as channel estimation, interactions with all upper layers and so on within a very short time to make the detection of each packet successfully. The slow parameter exchanges across different layers in traditional OSI model may not work properly in this sense due to the excessive latency in parameter exchanges in different layers. The Cross-Layer network design will help to shorten overall response time at the transceiver through Cross-Layer global optimization and thus will be in particular useful in $4 \mathrm{G}$ mobile networks design.

\subsubsection{Cross Layer Network Design}

The layered architecture is central to data network design. Layering provides the network design with modularity that facilitates standardization and implementation. In traditional OSI layer each layer is designed separately with control messages passing between adjacent layers. Cross-layer design can imply a joint design across all network layers simultaneously, which is desirable but highly complex. Alternately, it can entail choosing parameters or protocols at different network layers from existing designs in a joint fashion. Thus, the goal of Cross-layer design is to provide the best possible end to end performance of the application. Application examples include voice, web browsing, email, video sessions etc.

The need for Cross-layer network design is further motivated from the fact that the communication quality in future mobile networks should be dealt within an integrated and global fashion across the entire layered structure of a network. Hence, for such applications in Cross-layer design, for example, MAC protocol, the routing algorithm and the controller designs must adapt to the channel states. The key QoS parameters for multimedia communications in $4 \mathrm{G}$ mobile networks include transmission delay,

BER, packet drop rate, transmission rate, end-to-end throughput, etc. When the traffic contains very rich multimedia contents such as multi-party video conference sessions, the real time based QOS 
control over high speed data bursts in a future mobile network is an extremely complex process due to the need to cope with finding and mobility sensitive channel variations.

For such Multimedia applications Cross-layer network design, over the layers give significant performance gains when compared to the approaches with only layer-wise local optimization. In CrossLayer design, one of the most fundamental requirements on the physical layer design is its great elasticity or flexibility to act quickly on the demands from upper layer control. The change of transmission rate, spreading factor and other air-link parameters in 2$3 \mathrm{G}$ based systems is always difficult and costly, consuming a great amount of network-wise resources. Therefore, it is almost impossible to implement a Cross-layer optimal design based on existing platforms. For (eg) assume that a request for speeding up transmission rate comes from the application layer to fit some particular QoS and other service requirements.

The physical layer architecture acts on this request by replacing an existing long OVSF code with a shorter one thus with a lower SF.
To accomplish this task, the following actions should be taken in the network. If the shorter code is readily available in the cell of concern, the code is assigned to the application. In most cases, the rate requested from the application layer is not necessarily match exactly to the multiples of two. In this case, the punctured zeros should be inserted into the original data stream to fit the entire bandwidth. It is noted that a shorter code in OVSF code tree structure may likely block the other longer codes already in use.

In this case, cell-wise reshuffling of the code assignments likely happens for every such kind of rate change request. This incurs a lot of overhead traffic in both up and down links and affects almost all active mobile terminals in the cell, consuming a lot of air-link resource and time. Thus the elasticity of physical layer which is required for Cross-layer network design offers a great degree of freedom to the upper layer to control its operation. Thus, by adapting the parameters in Cross-Layer design, Multimedia application can be efficiently done in $4 \mathrm{G}$ Mobile networks.

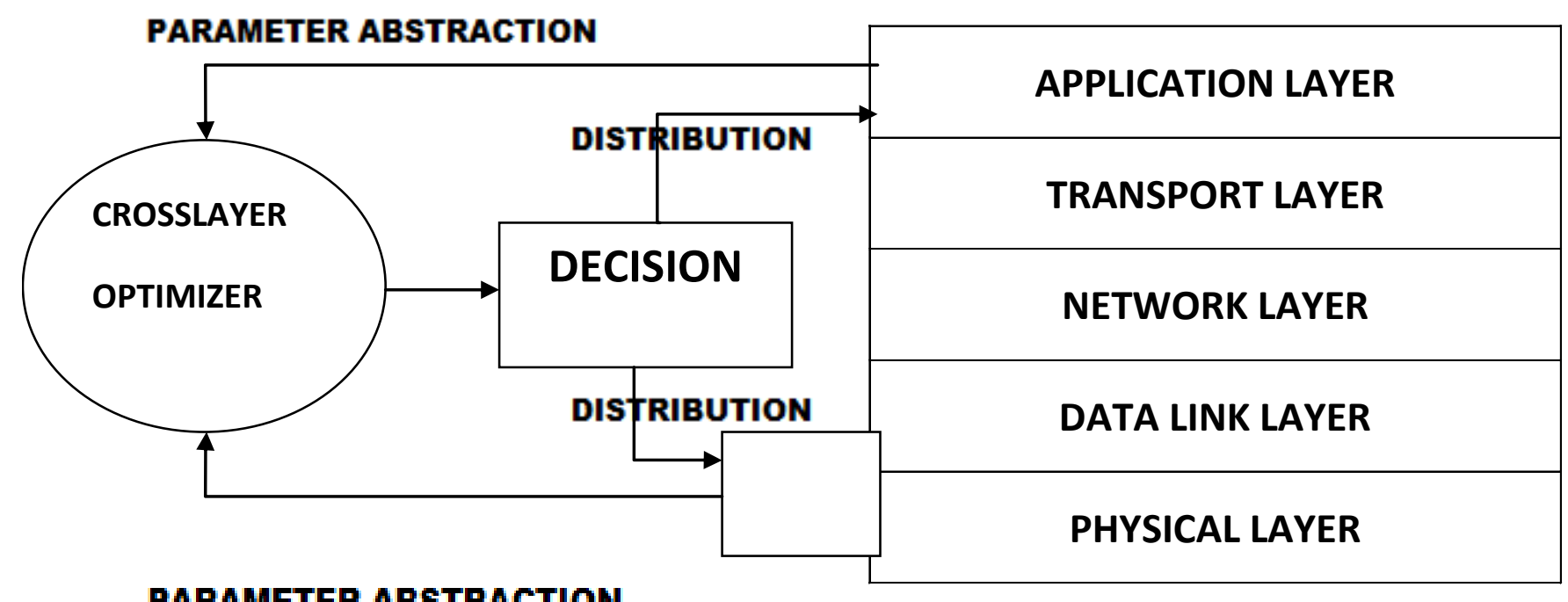

Fig 1. Conceptual model of Cross-Layer Feedback

\section{PAST AND ON GOING RESEARCH WORK IN QOS}

European Research Funded Projects, Two IST STREP,4 MORE-MC-CDMA Multiple- Antenna System On Chip for Radio Enhancements,IST-507039.The objective of 4 MORE is to research, develop and integrate and validate a cost effective low power system on chip solution for multi antenna multi carrier CDMA Mobile terminals based on joint optimization of layer 1 and layer 2.

PHOENIX,IST-001812 - PHOENIX - To develop a scheme offering the possibility to let the application world (source coding, ciphering) and the transmission world (channel coding, Modulation) talk to each other over an IPV6 protocol stack, so they can jointly develop an end to end optimized wireless communication link. NEWCOM, IST507325, NEWCOM Project E of IST Network of Excellence 
on Wireless Communication is to identify existing gaps in European Knowledge in cross-layer. To investigate potential benefits of cross-layer in wireless network design in relation to the methodology of separate layer design. Intends to consider the coupling of higher layers with physical layer and elaborate the information to be exploited from the physical layer to optimize network performance Defines and implements common software platforms realizing the agreed common frameworks/models to be successively integrated and maintained.

ERACLIN, MKTIK-2004-517518 ERACLIN-Enhanced Radio Resource Algorithms Based on Cross-Layer Issues for 4G Networks-Marie-Curie transfer knowledge project. Analysis of different cross-layer techniques to enhance the efficiency of wireless communication systems. Study of the potential benefits that can be obtained from using these techniques in different environments and in particular the explicit shift toward decentralized and adaptive MAC, radio resource management and routing approaches. Study of channel estimation information about the future channel state in the adaptability of all layers and overhead.

Reference [1] proposed an overview problems faced by all IP wireless mobile terminals (often referred to as $4 \mathrm{G}$ terminals). A simple framework for studying and solving these problems with Cross-Layer design has been presented. This framework first classifies known problems in four coordination planes - Security, QoS, Mobility and Wireless link. Security problem arise from multiple-layer encryption, which unnecessary power consumption and processing delay. QOS problems affect flows with QOS requirements and are caused by layer congestion control and link layer ARQ. The mobility problems are related to the effects of handover on transport layer connections and QoS signaling. Finally, wireless problems caused by packet corruption \& losses that are perceived by TCP as congestion indications causes poor performance. In order to help solve these problems, a simple interlayer coordination model was presented, consisting of a Cross-layer manager that receives event notifications from each protocol and perform management algorithms to control the internal state of each protocol and to correct their behavior as per information from other layers. However, the author has concluded that future work will include the development of management algorithms.

Reference [2] proposed the key NGMN design issues including architecture design, Cross-layer coordination, vertical hand-off \& admission control in a multi traffic environment. The proposals presented in this article provide valuable insight into the development of NGNM, but still a lot of work is necessary in areas of open research such as energy efficient multimode terminal development, session \& personal mobility consideration with terminal mobility, dimensioning of both the radio \& core network within the NGNM framework, Standardization of the inter working protocols and so forth. The ensuring proposal need to be designed is such a way that they reduce the amount of changes introduced into the existing systems \& promote interoperability within the hierarchical framework.

Reference [3] proposed to provide an overview of the cross-layer paradigm shift that is beginning to take place as wireless communication evolves from a circuit-switched infrastructure to a packet -based infrastructure. The advantages of Cross-layer networking appear to be real and will be increasingly important as capacity offered through the wireless LAN interface, approach the level of capacity that could be handled over the internet backbone. Based on observations, it appears that the above mentioned research areas are important for successful built out of future broadband wireless network. This paper provides viewpoint of the technical challenge \& emerging research areas that will lead to new network implementation to support the amalgamation of user services and QoS requirements.

Reference [4] proposed the survey of work addressing energy efficient and low-power design within all layers of the wireless network protocol stack. See [36] for the impact of higher order error statistics on the various layers of the protocol stack. The author discussed the impact of such information for adaptive protocol design throughout the stack. Power aware protocol in adhoc networks are discussed in [44]. Reference there in provide insight into the various power aware protocol proposals \& design issues. [46] present a conceptual framework for network \& client adaptation. They survey the various proposals for application adaptation \& map it to the conceptual framework. [47] present a survey on the various suggestion to improve TCP behavior over heterogeneous network.

Reference[5] In this paper, the problem of scheduling CBR traffic subject to deadline constraints was addressed, with the objective to reduce packet loss rate due to packet deadline expirations. The problem was studied in the context of (MDP) Markov Decision Process.

\subsection{Possible Approaches on QoS Enhancement in $4 G$ Networks Status Identified from Literature}

To support multimedia traffic with QoS - IMS [IP Multimedia subsystem] could be used along with the below mentioned parameters.

SDR Software Defined Radio to provide access to network independent services.[7], IPV6 forms common platform for $4 \mathrm{G}$ networks. To achieve higher data rate, modification of radio and core network could be done to enable new / emerging networks for seamless connectivity to the general framework [9].Cross-Layer coordination among different layers is facilitated through well defined message interfaces such as API [Application Programming Interface], ISI [Inter Signaling Pipe] and ICMP. A common control/signaling mechanism could be utilized to assist access network discovery, location management \& vertical handoff by periodically computing \& broadcasting a list of available radio access network (RANs). List of surrounding BS IDs, their associated AR IDs \& network alternatives \& their QoS parameters. However, the common signaling problem could be resolved using an overlay structure on existing BS / APS (as proposed by MIRAI).

For resource management, a distributed BB (bandwidth broker) is proposed inside each router domain, along with backup facility BB includes SLA, SLS, ACS, A\&A, PDP[4]. Specifically Cross-layer coordination for Application \& Network layers only could be provided by using separate external servers for time critical services. In $4 \mathrm{G}$ to provide always connectivity at anytime along with horizontal handoff, forced vertical hand-off was also proposed to upgrade application QoS against dropped sessions. To be specific, Periodical Vertical handoff algorithm could be used. This algorithm can be embedded within terminal architecture for MT controlled (Mobile Terminal) against MT assisted handoffs as in 3G.[11].

Problems pertaining to varying network conditions such as latency, bandwidth \& energy could be rectified using watermark based mechanism along with ICMP messages in 
Cross-layer coordination. 4G deals with heterogonous networks, so for access network selection, the proposed method will be AHP [Analytic Hierarchy Process] and GRA [Grey Relational Analysis] taking throughput, delay, jitter, reliability, BER, burst error, average retransmissions, security, cost \& power consumption [15]. For QoS normalization, the proposed algorithm takes smaller - the better concept (timeliness, reliability, cost \& power consumption). Likewise larger - the better will be referred for throughput $\&$ security.

To get fair CAC algorithm [Call Admission Control] in a multi traffic environment, adaptive Markov - model based CAC policy is proposed. For testing advanced mobile services, based on NTP - SIOT [Service Interoperability Test] TTCN-3 [Taiwan] platform could be used as test benches for OMA [Open Mobile Alliance] multimedia messaging service exclusively for 4G. TTCN [Testing \& Test Control Notation] simulations tools such as NS-2, MATLAB, Glomosim, etc could be used along with embedded system language used for testing is IDL [Interface Definition Language] and JAVA.

\section{CONCLUSION}

This paper is based on the survey status of QoS in $4 \mathrm{G}$ networks to provide all time connectivity with minimal resource amidst the stringent bandwidth requirements particularly for multimedia data. $4 \mathrm{G}$ as defined will be the future network with appreciable data rate of more than $10 \mathrm{Mbps}$ with seamless connectivity equal to the wired QoS status. Thus, in future by adapting techniques in tandem, of QoS parameter in CLD [Cross-Layer Design] optimization will be achieved regarding achievable QoS in 4G networks.

\section{REFERENCES}

[1] Gustavo Carnerio, Jose Ruela and Manuel Ricardo, "Cross-Layer Design in 4G Wireless Terminals", IEEE Wireless Communication, April 2004, page 7-13

[2] M.Rubaiyat Kibria \& Abbas Jamalipour, "On Designing Issues of the NGMN", IEEE Networks, Jan/Feb 2007, Vol21, No1 page 54-57.

[3] SanjayShakkotai, Theodore S.Rappaport \& Peter C. Karlson, "Cross-Layer Design for Wireless Networks", IEEE Wireless Communication, June 2003, page 2-14.

[4] Viyay.T.Raisinghani \& Sridhar Iyer, "Cross-Layer Design Optimization in Wireless Protocol Stacks", Elsevier Science, March 2003
[5] Tianmin Ren, Iordanis Koutsopoulos7Lean drosTassiulus, "QOS Provisioning for Real Time traffic in Wireless Packet Networks", IEEE GLOBECOM 2002.

[6] LodewukvanHoesel, Tim Nieberg, Jian $\mathrm{Wu}$, PaulJ.M.Havinga, "Prolonging the lifetime of wireless sensor networks by cross-layer interaction", IEEE Wireless Communication, Dec 04, Vol.11. No.6, pp 6165 .

[7] Vikas- Kawadia \& P.R.Kumar, "A cautionary Perspective on cross-layer design", IEEE Wireless Communications, Feb 05, Vol.12.No.1, pp 57-62.

[8] IEEE Vechicular Technology, Sep 06, Vol.1, No.3

[9] CorneliaKappler, Petteri poyhonen, MartinJohnsson, Stefan Schmid, "Dynamic Network Composition for beyond 3G Networks: A 3GPP viewpoint", IEEE Network, Jan/Feb 07, Vol.21, No.1, pp 74-77.

[10] Bader Al-Manthari, Hossam Hassanein, Nidal Nasser, Schmid, "Packet Scheduling in $3.5 \mathrm{G}$ High - Speed Downlink Packet Access Networks", IEEE Network, Jan/Feb 07, Vol.21, No.1, pp 52-57.

[11] Implementable Wireless Access for B3G Networks, IEEE Communication, March 07, Vol.45, No.3, Jongmin Jeong, Zygmunt J.Hass, "An Integrated Security Frame work for OWN Architecture", IEEE Wireless Communication, April 07, Vol.14,No.2.

[12] Zhikui Chen,"A Customizable QOS Strategy for Convergent Heterogeneous Wireless Communication", IEEE Wireless Communication April 07, Vol.14, No.2.

[13] Chen Yiping, Yang Yuhang, "A New 4G ArchitectureProviding Multimode Terminals Always Best Connected Services", IEEE Wireless Communication April 07, Vol.14, No.2, pp 33-39.

[14] Young-JuneChoi, KwangBok Lee, Saewoong Bahk, "All- IP 4G Network Architecture for Efficient Mobility and Resource Management", IEEE Wireless Communication April 07, Vol.14, No.2, pp 76-81.

[15] YoujunGao, Hui Tian, Lei Sun, Haibo Xu, "Research on the Access Network and MAC Technique form Beyond3G Systems", IEEE Wireless Communication April 07, Vol.14, No.2, pp67-73. 